\title{
A MATRIX HARNACK ESTIMATE FOR THE HEAT EQUATION
}

\author{
RICHARD S. HAMILTON
}

In the important paper[LY] by Peter Li and S.-T. Yau, they show how the classical Harnack principle for the heat equation on a manifold can be derived from a differential inequality. In particular, they show that for any positive solution $f>0$ of the heat equation

$$
\frac{\partial f}{\partial t}=\Delta f
$$

on a compact Riemannian manifold of dimension $m$ solving the equation for $t>0$, if the manifold has weakly positive Ricci curvature $R_{i j} \geq 0$ then for any vector field $V$ on $M$

$$
\frac{\partial f}{\partial t}+\frac{1}{2 t} f+2 D f(V)+f|V|^{2} \geq 0 ;
$$

a similar result holds with an error term if the Ricci curvature is bounded below. The quadratic version in $V$ given here is equivalent to the more complicated formula in their paper by choosing the optimal $V$. We shall show in this paper that the Harnack estimate of $\mathrm{Li}$ and Yau is the trace of a full matrix inequality.

Main Theorem. If $M$ is a compact Riemannian manifold and $f>0$ is a positive solution to the heat equation on $M$

$$
\frac{\partial f}{\partial t}=\Delta f
$$

for $t>0$, then for any vector field $V_{i}$ on $M$ we have

$$
D_{i} D_{j} f+\frac{1}{2 t} f g_{i j}+D_{i} f \cdot V_{j}+D_{j} f \cdot V_{i}+f V_{i} V_{j} \geq 0
$$

Research partially supported by NSF contract DMS 90-03333. 
provided $M$ is Ricci parallel and has weakly positive sectional curvatures; while in general we can find constants $B$ and $C$ depending only on the geometry of $M$ (in particular the diameter, the volume, the curvature and the covariant derivative of the Ricci curvature) such that $t^{m / 2} f \leq B$ and for all $V_{i}$ $D_{i} D_{j} f+\frac{1}{2 t} f g_{i j}+D_{i} f \cdot V_{j}+D_{j} f \cdot V_{i}+f V_{i} V_{j}+C f\left[1+\log \left(B / t^{m / 2} f\right)\right] g_{i j} \geq 0$.

This inequality is equivalent to Theorem 4.3 by choosing the optimal $V_{i}=$ $-D_{i} f / f$. The result can also be proved for bounded positive solutions on complete manifolds with bounded curvature and derivatives (we omit this technical detail). One can easily check that the inequality becomes an equality for the fundamental solution on Euclidean space $R^{m}$

$$
f=\frac{1}{t^{m / 2}} e^{-|x|^{2} / 4 t}
$$

which is a homothetically expanding soliton along the vector field $V_{i}=x_{i} / 2 t$. The hypothesis that $M$ is Ricci flat with weakly positive sectional curvatures is satisfied on a torus or a sphere or a complex projective space, or a product of such, or a quotient of a product by a finite group of isometries. In this case the Harnack estimate becomes

$$
D_{i} D_{j} \log f+\frac{1}{2 t} g_{i j} \geq 0
$$

which is a kind of logarithmic convexity. In particular, along any geodesic $x(s)$ para-metrized by arc length $\theta$ we have

$$
\frac{d^{2}}{d s^{2}} \log f+\frac{1}{2 t} \geq 0
$$

and hence

$$
\log f+\frac{s^{2}}{4 t}
$$

is convex; from which we conclude that along any geodesic $x(s)$

$$
f(x(0), t) \leq f(x(s), t)^{1 / 2} f(x(-s), t)^{1 / 2} e^{s^{2} / 4 t} .
$$

Also if $f$ has its maximum along the geodesic $x(s)$ at $x=0$ at time $t$ then everywhere else on the geodesic

$$
f(x(s), t) \geq f(x(0), t) e^{-s^{2} / 4 t} .
$$


Similar results with small error terms can be derived for manifolds $M$ with general curvature. The Li-Yau result allows comparisons between different points at different times; the matrix result allows comparisons between different points at the same time also. However, their result is not entirely contained in ours, in the sense that for general geometries the trace result can be obtained with restrictions only on the Ricci curvature.

The matrix Harnack estimate given here is very useful for proving monotonicity formulas, as in $[\mathrm{GH}]$. We also include in this paper several estimates on positive solutions of the heat equation which are used in that paper, for which this one is intended to serve as a general reference.

1.

Let $M$ be a compact manifold of dimension $m$ and let $f$ be a smooth solution of the heat equation on $0 \leq t<\infty$

$$
\frac{\partial f}{\partial t}=\Delta f
$$

with $f>0$ for all time. We shall derive estimates on $f$ that provide information on its behavior. The first is a derivative bound.

Theorem 1.1. Let $f$ be a positive solution with $f \leq A$, and let $K$ be a lower bound on the Ricci curvatures of $M$, so that $R_{i j} \geq-K g_{i j}$ with $K \geq 0$. Then

$$
t|D f|^{2} \leq(1+2 K t) f^{2} \log (A / f) .
$$

Proof. We compute

$$
\frac{\partial}{\partial t} \frac{|D f|^{2}}{f}=\Delta \frac{|D f|^{2}}{f}-\frac{2}{f}\left|D_{i} D_{j} f-\frac{D_{i} f D_{j} f}{f}\right|^{2}-2 R_{i j} \frac{D_{i} f D_{j} f}{f}
$$

and

Now if

$$
\frac{\partial}{\partial t} f \log (A / f)=\triangle\left(f \log \frac{A}{f}\right)+\frac{|D f|^{2}}{f} .
$$

$$
\varphi=\frac{t}{1+2 K t}
$$

then

$$
\frac{d}{d t} \varphi+2 K \varphi \leq 1
$$


If we put

$$
h=\varphi \frac{|D f|^{2}}{f}-f \log (A / f)
$$

then $h \geq 0$ at $t=0$ and

$$
\frac{\partial h}{\partial t} \leq \Delta h
$$

as we see from the previous computations, throwing away the square and bounding the Ricci curvature term with $R_{i j} \geq-K g_{i j}$. It follows from the maximum principle that $h \leq 0$ for all time, and this is the theorem.

We can use this result to get an upper bound on the solution $f$ just in terms of its average value and the elapsed time.

Corollary 1.2. Let $f$ be a positive solution to the heat equation. Then

$$
f(x, t) \leq \frac{B}{t^{m / 2}} \int_{M} f(x, t) d x
$$

for $0<t \leq 1$ with a constant $B$ depending only on $M$.

Proof. Consider the point $(\xi, \tau)$ in $0 \leq t \leq 1$ where $t^{m / 2} f(x, t)$ assumes its maximum value, which we call $b$. Then

$$
\max t^{m / 2} f(x, t)=\tau^{m / 2} f(\xi, \tau)=b .
$$

On the restricted time interval $\tau / 2 \leq t \leq \tau$ we have $f(x, t) \leq A$ where $A=2^{m / 2} b / \tau^{m / 2}$. Applying Theorem 1.1 on this time interval

$$
\frac{\tau}{2}|D f(x, \tau)|^{2} \leq(1+K \tau) f(x, \tau)^{2} \log (A / f(x, \tau))
$$

for all $x \in M$. Let

$$
\ell(x, \tau)=\log (A / f(x, \tau))
$$

and conclude that

$$
|D \sqrt{\ell}(x, \tau)| \leq \sqrt{\frac{1+K \tau}{2 \tau}}
$$

If we let

$$
\rho=\sqrt{\frac{\tau}{1+K \tau}}
$$

then for all $x$ in the ball $d(x, \xi) \leq \rho$ we have

$$
\sqrt{\ell}(x, \tau) \leq \sqrt{\ell}(\xi, \tau)+1 / \sqrt{2}
$$


Also we have

$$
\ell(\xi, \tau)=\log (A / f(\xi, \tau))=\frac{m}{2} \log 2
$$

which shows that

$$
\ell(x, \tau) \leq C_{m}
$$

for all $x$ in the ball $d(x, \xi) \leq \rho$, where $C_{m}$ denotes a constant depending only on $m$. Consequently

$$
f(x, \tau) \geq C_{m} A
$$

where $C_{m}>0$ is another constant depending only on $m$. We integrate this estimate over the ball $d(x, \xi) \leq \rho$. Since $\rho \approx \sqrt{\tau}$ for small $\tau$, the ball will have a volume

$$
V \geq C_{M} \tau^{m / 2}
$$

for some constant $C_{M}>0$ depending only on $M$ as long as $\tau \leq 1$. This gives

$$
C_{M} \tau^{m / 2} A \leq \int_{M} f(x, \tau) d x
$$

Since the integral of $f$ over $M$ is conserved, this proves the corollary.

We can now use this bound in the original estimate.

Corollary 1.3. There exists constants $B$ and $C$ depending only on $M$, such that if $f$ is any positive solution of the heat equation with $\int f=1$, then for $0<t \leq 1$

$$
t|D f|^{2} \leq C f^{2} \log \left(B / t^{m / 2} f\right)
$$

Proof. To estimate $|D f|^{2}$ at any time $\tau$, we apply Theorem 1.1 on $\tau / 2 \leq t \leq \tau$ where we use the bound from Corollary 1.2.

2.

Next we prove a Harnack estimate similar to that in the paper of $\mathrm{Li}$ and Yau[LY]. We need the case of negative curvature.

Theorem 2.1. Let $f$ be a positive solution of the heat equation on a compact manifold $M$ whose Ricci curvature is bounded below by $R_{i j} \geq-K g_{i j}$ for $K \geq 0$. Then

$$
\frac{\partial f}{\partial t}-e^{-2 K t} \frac{|D f|^{2}}{f}+e^{2 K t} \frac{m}{2 t} f \geq 0
$$


Proof. We use

$$
\frac{\partial}{\partial t} \frac{|D f|^{2}}{f}=\Delta \frac{|D f|^{2}}{f}-\frac{2}{f}\left|D_{i} D_{j} f-\frac{D_{i} f D_{j} f}{f}\right|^{2}-2 R_{i j} \frac{D_{i} f D_{j} f}{f}
$$

and estimate

$$
\left|D_{i} D_{j} f-\frac{D_{i} f D_{j} f}{f}\right|^{2} \geq \frac{1}{m}\left(\frac{\partial f}{\partial t}-\frac{|D f|^{2}}{f}\right)^{2}
$$

from the trace. When

$$
h=\frac{\partial f}{\partial t}-e^{-2 K t} \frac{|D f|^{2}}{f}+e^{2 K t} \frac{m}{2 t} f
$$

this gives

$$
\frac{\partial h}{\partial t} \geq \Delta h+\frac{2}{m f} e^{-2 K t}\left(\frac{\partial f}{\partial t}-\frac{|D f|^{2}}{f}\right)^{2}-e^{2 K t} \frac{m}{2 t^{2}} f
$$

where we use the term from the time derivative falling on $e^{-2 K t}$ to cancel the term with the Ricci curvature, and throw away the term from the time derivative falling on $e^{2 K t}$. Now when $h \leq 0$ we have

$$
0 \leq e^{2 K t} \frac{m}{2 t} f \leq e^{-2 K t} \frac{|D f|^{2}}{f}-\frac{\partial f}{\partial t} \leq \frac{|D f|^{2}}{f}-\frac{\partial f}{\partial t}
$$

and hence

$$
\frac{\partial h}{\partial t} \geq \Delta h \quad \text { when } \quad h \leq 0 .
$$

But $h \rightarrow+\infty$ as $t \rightarrow 0$, so $h \geq 0$ for all time by the maximum principle.

Corollary 2.2. If $f$ is a positive solution to the heat equation for $t \geq 0$ and $-K$ is a lower bound for the Ricci curvature then for any points $\xi$ and $X$ in $M$ and any times $\tau$ and $T$ with $0<\tau<T$ we have

$$
\begin{aligned}
& f(\xi, \tau) \leq \\
& \left(\frac{T}{\tau}\right)^{m / 2} f(X, T) \exp \left\{\frac{1}{4} e^{2 K \tau}[1+2 K(T-\tau)] \frac{d(X, \xi)^{2}}{T-\tau}+\frac{m}{2}\left[e^{2 K T}-e^{2 K \tau}\right]\right\} .
\end{aligned}
$$

Proof. Let $\ell=\log f$. The previous theorem says

$$
\frac{\partial \ell}{\partial t}-e^{-2 K t}|D \ell|^{2}+e^{2 K t} \frac{m}{2 t} \geq 0 .
$$


Choose a path $x=x(t)$ with $x=\xi$ at $t=\tau$ and $x=X$ at $t=T$. Then integrating along the path

$$
\ell(X, T)-\ell(\xi, \tau)=\int_{\tau}^{T}\left\{\frac{\partial \ell}{\partial t}+D \ell \frac{d x}{d t}\right\} d t
$$

and if we use the previous estimate and

$$
e^{-2 K t}|D \ell|^{2}+D \ell \frac{d x}{d t} \geq-\frac{1}{4} e^{2 K t}\left|\frac{d x}{d t}\right|^{2}
$$

we get

$$
\ell(X, T)-\ell(\xi, \tau) \geq-\frac{1}{4} \int_{\tau}^{T} e^{2 K t}\left|\frac{d x}{d t}\right|^{2} d t-\int_{\tau}^{T} \frac{m}{2 t} e^{2 K t} d t
$$

Now we choose the path from $\xi$ to $X$ to lie along a geodesic, but with arc length

$$
s=a\left[e^{-2 K \tau}-e^{-2 K t}\right]
$$

where $a$ is a constant. In order to start at $\xi$ with $s=0$ at $t=\tau$ and end at $X$ with $s=d(X, \xi)$ at $t=T$ we need

$$
d(x, \xi)=a\left[e^{-2 K \tau}-e^{-2 K t}\right]
$$

This makes

$$
\int_{\tau}^{T} e^{2 K t}\left|\frac{d x}{d t}\right|^{2} d t=\frac{2 K}{e^{-2 K \tau}-e^{-2 K T}} d(X, \xi)^{2} .
$$

To make this look better, we estimate

$$
e^{u} \geq 1+u
$$

which makes

$$
\frac{1}{1-e^{-u}} \leq \frac{1+u}{u}
$$

and

$$
\frac{2 K}{e^{-2 K \tau}-e^{-2 K T}}=\frac{2 K e^{2 K \tau}}{1-e^{-2 K(T-\tau)}} \leq \frac{e^{2 K \tau}[1+2 K(T-\tau)]}{T-\tau} .
$$

Finally since

$$
\frac{e^{u}}{u} \leq \frac{1}{u}+e^{u}
$$

we can estimate

$$
\int_{\tau}^{T} \frac{e^{2 K t}}{t} d t \leq \log \left(\frac{T}{\tau}\right)+\left(e^{2 K T}-e^{2 K \tau}\right) .
$$


Thus

$$
\begin{aligned}
\ell(\xi, \tau) \leq & \ell(X, T)+\frac{1}{4} e^{2 K \tau}[1+2 K(T-\tau)] \frac{d(X, \xi)^{2}}{T-\tau} \\
& +\frac{m}{2} \log \left(\frac{T}{\tau}\right)+\frac{m}{2}\left(e^{2 K T}-e^{2 K \tau}\right) .
\end{aligned}
$$

If we exponentiate this we get the corollary.

We can use this estimate to get a good lower bound on the fundamental solution of the heat equation.

Corollary 2.3. The fundamental solution of the heat equation $k(x, t, y)$ satisfies

$$
k(x, t, y) \geq \frac{c}{t^{m / 2}} \exp -\left\{\frac{1}{4}(1+2 K t) \frac{d(x, y)^{2}}{t}+\frac{m}{2} e^{2 K t}\right\}
$$

for some constant $c$ depending only on $M$.

Proof. It is well known that

$$
k(x, t, x) \geq c / t^{m / 2} .
$$

We apply this in the previous estimate taking $f(x, t)=k(x, t, y)$ and putting $\xi=y$ and letting $\tau \rightarrow 0$.

3.

We are now in a position to establish a basic comparison result that we need for the $\varepsilon$-regularity result.

Theorem 3.1. For any $\delta>0$ and any constant $C>1$ and for any $X \in M$ and any $T$ with $0<T \leq 1$, there exists a $\rho>0$ such that if $\xi \in M$ satisfies $d(X, \xi) \leq \rho$ and $\tau$ satisfies $T-\rho^{2} \leq \tau \leq T$ then for any positive solution $f(x, t)$ of the heat equation with $\int f=1$

$$
\tau f(\xi, \tau) \leq \delta+C T f(X, T) .
$$

Proof. First note by Corollary 2.2 that

$$
f(\xi, \tau) \leq\left(\frac{T}{\tau}\right)^{m / 2} f(\xi, T) \exp \left\{\frac{m}{2}\left[e^{2 K T}-e^{2 K \tau}\right]\right\}
$$

and hence if $\rho$ is small enough

$$
f(\xi, \tau) \leq C f(\xi, T)
$$


for any $C>1$ we desire. Thus it suffices to prove the estimate when $T=\tau$. Thus we must show

$$
f(\xi, T) \leq \frac{\delta}{T}+C f(X, T) .
$$

For this we use the gradient estimate in Corollary 1.3, with $t=T$, so that

$$
T|D f(x, T)|^{2} \leq K f(x, T)^{2} \log \left(B / T^{m / 2} f(x, T)\right)
$$

for all $x$. Let

$$
\ell=\log \left(B / T^{m / 2} f(x, T)\right)
$$

and observe

$$
T|D \ell|^{2} \leq K \ell
$$

and hence

$$
|D \sqrt{\ell}| \leq \frac{1}{2} \sqrt{\frac{K}{T}}
$$

This makes

$$
\sqrt{\ell}(X, T) \leq \sqrt{\ell}(\xi, T)+\frac{1}{2} \rho \sqrt{\frac{K}{T}} .
$$

If $f(\xi, T) \leq \delta T$, we are done, otherwise

$$
\ell(\xi, T) \leq \log \left(\frac{B}{\delta T^{\frac{m}{2}-1}}\right) .
$$

Squaring the previous inequality gives

$$
\ell(X, T) \leq \ell(\xi, T)+\rho \sqrt{\frac{K}{T} \log \left(\frac{B}{\delta T^{m 2-1}}\right)}+\frac{1}{4} \rho^{2} \frac{K}{T} .
$$

By choosing $\rho$ small enough, we can make term $2+$ term $3 \leq \eta$ for any $\eta>0$.

For such a choice of $\rho$ we have

$$
\begin{aligned}
\ell(X, T) & \leq \ell(\xi, T)+\eta \\
\Rightarrow \quad f(\xi, T) & \leq e^{\eta} f(X, T) \leq \frac{\delta}{T}+C f(X, T)
\end{aligned}
$$

with $e^{\eta}=C>1$. This proves the theorem. 
4.

Finally we come to the second derivative estimate. First we need a bound on $\triangle f$.

Lemma 4.1. There exists a constant $C$ depending only on $M$ such that if $f$ is a positive solution of the heat equation with $0<f \leq A$ then for $0 \leq t \leq 1$

$$
t \triangle f \leq C f[1+\log (A / f)] .
$$

Proof. Again we use

$$
\frac{\partial}{\partial t} \frac{|D f|^{2}}{f} \leq \Delta \frac{|D f|^{2}}{f}-\frac{2}{m f}\left[\Delta f-\frac{|D f|^{2}}{f}\right]^{2}+2 K \frac{|D f|^{2}}{f}
$$

where $-K$ is a lower bound on the Ricci curvature. This time we let $\varphi=$ $\left[e^{K t}-1\right] / K e^{K t}$ and let $h=\varphi\left[\triangle f+|D f|^{\frac{2}{f}}\right]-f[m+4 \log (A / f)]$ and compute

$$
\frac{\partial h}{\partial t} \leq \Delta h--\frac{2 \varphi}{m f}\left[\Delta f-\frac{|D f|^{2}}{f}\right]^{2}+\varphi^{\prime}\left[\Delta f-\frac{|D f|^{2}}{f}\right]-2 \frac{|D f|^{2}}{f}
$$

using $\varphi^{\prime}+K \varphi=1$. Now we claim that

$$
\frac{\partial h}{\partial t} \leq \Delta h \quad \text { whenever } \quad h \geq 0
$$

and we see this by examining three cases.

(i) If $\Delta f \leq \frac{|D f|^{2}}{f}$ we are done, since $\varphi^{\prime} \geq 0$.

(ii) If $\frac{|D f|^{2}}{f} \leq \Delta f \leq 3 \frac{|D f|^{2}}{f}$ we are also done, since $\varphi^{\prime} \leq 1$.

(iii) If $3 \frac{|D f|^{2}}{f} \leq \Delta f$, then when $h \geq 0$

$$
2\left[\Delta f-\frac{|D f|^{2}}{f}\right] \geq \Delta f+\frac{|D f|^{2}}{f} \geq \frac{m f}{\varphi}
$$

and since $\varphi^{\prime} \leq 1$ we are done completely.

Since $h \leq 0$ at $t=0$, the maximum principle implies $h \leq 0$ for all $t$. Since $e^{u}-1 \geq u$, it follows from the definition of $\varphi$ that $t \leq e^{K t} \varphi(t) \leq e^{K} \varphi(t)$ for $0 \leq t \leq 1$, and the result follows. 
Corollary 4.2. There exist constants $B$ and $C$ depending only on $M$ such that if $f$ is a positive solution to the heat equation with $\int f \leq 1$ then for $0<t \leq 1$

$$
t \triangle f \leq C f\left[1+\log \left(B / t^{m / 2} f\right)\right] .
$$

Proof. We know that for some constant $B$ we have $t^{m / 2} f \leq B$ by Corollary 1.2. Apply Lemma 4.1 on the time interval $\tau / 2 \leq t \leq \tau$ using the bound $f \leq 2^{m / 2} B / \tau^{m / 2}=A$ and we get the desired conclusion.

Theorem 4.3. Let $M$ be a compact manifold. There exist constants $B$ and $C$ depending only on $M$ such that if $f$ is a positive solution to the heat equation with $\int f \leq 1$ then for $0<t \leq 1$ we have

$$
D_{i} D_{j} f-\frac{D_{i} f D_{j} f}{f}+\frac{1}{2 t} f g_{i j}+C f\left[1+\log \left(B / t^{m / 2} f\right)\right] g_{i j} \geq 0 .
$$

Proof. Let

$$
H_{i j}=D_{i} D_{j} f-\frac{D_{i} f D_{j} f}{f} .
$$

Then in a straightforward manner we compute

$$
\begin{aligned}
\frac{\partial}{\partial t} H_{i j}= & \triangle H_{i j}+\frac{2}{f} H_{i j}^{2}+2 R_{i k j l} H_{k l}-R_{i k} H_{j k}-R_{j k} H_{i k} \\
& +\frac{2}{f} R_{i k j l} D_{k} f D_{l} f+\left[D_{l} R_{i j}-D_{i} R_{j l}-D_{j} R_{i l}\right] D_{l} f
\end{aligned}
$$

where $H_{i j}^{2}=H_{i k} H_{j k}$ is the square of the matrix. Let

$$
\ell=m+\log \left(B / t^{m / 2} f\right)
$$

where $B$ is a constant with $t^{m / 2} f \leq B$ from Corollary 1.2, and let

$$
P=\frac{1}{2 t} f+A\left(K+L^{2 / 3}\right) f \ell
$$

where $-K$ is a lower bound for the sectional curvature of $M, L$ is an upper bound for $\left|D_{i} R_{j k}\right|$ and $A$ is a constant to be determined later. We compute

$$
\frac{\partial P}{\partial t}=\triangle P+Q
$$

where

$$
Q=-\frac{1}{2 t^{2}} f-\frac{m A}{2 t}\left(K+L^{2 / 3}\right) f+A\left(K+L^{2 / 3}\right) \frac{|D f|^{2}}{f} .
$$


Note that

$$
\frac{2}{f} P^{2} \geq \frac{1}{2 t^{2}} f+\frac{2 A}{t}\left(K+L^{2 / 3}\right) f \ell+2 A^{2}\left(K+L^{2 / 3}\right)^{2} f \ell^{2}
$$

and since $\ell \geq m$ we get

$$
\frac{2}{f} P^{2}+Q \geq \frac{A}{t}\left(K+L^{2 / 3}\right) f \ell+2 A^{2} m\left(K+L^{2 / 3}\right)^{2} f \ell .
$$

Now let

$$
N_{i j}=H_{i j}+P g_{i j}
$$

and compute

$$
\begin{aligned}
\frac{\partial}{\partial t} N_{i j}= & \Delta N_{i j}+\frac{2}{f} N_{i j}^{2}-\frac{4}{f} P N_{i j} \\
& +2 R_{i k j l} N_{k l}-R_{i k} N_{j k}-R_{j k} N_{i k} \\
& +\frac{2}{f} R_{i k j l} D_{k} f D_{l} f+\left[D_{\ell} R_{i j}-D_{i} R_{j \ell}-D_{j} R_{i \ell}\right] D_{\ell} f \\
& +\left[\frac{2}{f} P^{2}+Q\right] g_{i j} .
\end{aligned}
$$

If the sectional curvatures of $M$ are bounded below by $-K$, then

$$
P_{i k j l}=R_{i k j l}+K\left(g_{i j} g_{k l}-g_{i l} g_{j k}\right)
$$

is positive in the sense that

$$
P_{i k j \ell} v_{i} w_{k} v_{j} w_{\ell} \geq 0
$$

for all vectors $v$ and $w$. This makes

$$
R_{i k j \ell} N_{k \ell} \geq P_{i k j \ell} N_{k \ell}-K N g_{i j}+K N_{i j}
$$

where $N$ is the trace of $N_{i j}$. Also we have

$$
R_{i k j l} D_{k} f D_{\ell} f \geq-K|D f|^{2} g_{i j}
$$

and if $\left|D_{i} R_{j k}\right| \leq L$ then

$$
\left[D_{\ell} R_{i j}-D_{i} R_{j \ell}-D_{j} R_{i \ell}\right] D_{\ell} f \geq-3 L|D f| g_{i j} .
$$

Also we can bound

$$
L|D f| \leq L^{2 / 3} \frac{|D f|^{2}}{f}+L^{4 / 3} f
$$


Assembling these estimates, we get

$$
\begin{aligned}
\frac{\partial}{\partial t} N_{i j} \geq & \triangle N_{i j}+\frac{2}{f} N_{i j}^{2}-\frac{4}{f} P N_{i j} \\
& +2 P_{i k j l} N_{k l}+2 K N_{i j}-R_{i k} N_{j k}-R_{j k} N_{i k} \\
& +Z g_{i j}
\end{aligned}
$$

where

$$
Z=\frac{2}{f} P^{2}+Q-2 K N-\left(2 K+3 L^{2 / 3}\right) \frac{|D f|^{2}}{f}-3 L^{4 / 3} f
$$

Now observe that

$$
N=\Delta f-\frac{|D f|^{2}}{f}+m\left[\frac{1}{2 t} f+A\left(K+L^{2 / 3}\right) f \ell\right] .
$$

By Corollary 4.2 we have a bound

$$
\triangle f \leq \frac{C}{t} f \ell
$$

for some constant $C$ (and the same $B$ ). We also have

$$
\frac{|D f|^{2}}{f} \leq \frac{C}{t} f \ell
$$

for some constant $C$ from Corollary 1.3. Remembering $\ell \geq m$, this makes

$$
2 K N+\left(2 K+3 L^{2 / 3}\right) \frac{|D f|^{2}}{f}+3 L^{4 / 3} f \leq \frac{C}{t}\left(K+L^{2 / 3}\right) f \ell+C A\left(K+L^{2 / 3}\right) f \ell
$$

for some constant $C$ depending only on $M$. Now if we choose $A$ large enough with respect to $C$, we can make $Z \geq 0$. Then the maximum principle for matrices (see Hamilton[H]) implies that $N_{i j} \geq 0$ for all time. We only have to check that $N_{i j} \geq 0$ at $t=0$, and that each of the terms

$$
N_{i j}^{2}, P N_{i j}, P_{i k j l} N_{k l}, K N_{i j}, R_{i k} N_{j k}+R_{j k} N_{i k}
$$

is non-negative at a null-eigenvector of $N_{i j}$. This is immediate except perhaps for $P_{i k j l} N_{k l}$, but here it is also clear if we diagonalize $N_{k l}$ with respect to a basis. This completes the proof.

Corollary 4.4. Suppose $M$ has non-negative sectional curvature and is Ricci parallel, so that $R_{i k j l} \geq 0$ and $D_{i} R_{j k}=0$. Then for any positive solution $f$ of the heat equation

$$
D_{i} D_{j} f-\frac{D_{i} f D_{j} f}{f}+\frac{1}{2 t} f g_{i j} \geq 0
$$


Proof. The above proof simplifies, taking $K=L=0$.

This result applies on flat tori, on spheres, on complex projective spaces, on a product of such spaces, and on a quotient of a product.

\section{REFERENCES}

[GH] Grayson, M. and Hamilton, R. S., The formation of singularities in the harmonic map heat flow, preprint, UCSD.

[H] Hamilton, R. S., Four-manifolds with positive curvature operator, J. Differential Geom. 24 (1986), 153-179.

[LY] Li, P., and Yau, S.-T., On the parabolic kernel of the Schrödinger operator. Acta Math. 156 (1986), 153-201.

University of California, San Diego, U. S. A.

ReCeived July 10, 1992 